\title{
Effects of epidermal growth factor receptor fusion protein on the cytotoxic activity of SOCS1-silenced dendritic cells in vitro
}

\author{
QICHUAN JIANG ${ }^{1}$, XUEFENG WANG $^{2}$, MING QIAN $^{3}$, DONG CHEN $^{1}$ and YANAN XU ${ }^{1}$ \\ ${ }^{1}$ Department of Otolaryngology Head and Neck Surgery, The First Affiliated Hospital of Liaoning Medical University; \\ ${ }^{2}$ Department of Otolaryngology Head and Neck Surgery, Liaoning Medical University, Jinzhou, Liaoning 121000, P.R. China; \\ ${ }^{3}$ Department of Health Sciences Center, University of Oklahoma Health Sciences Center, Oklahoma City, OK 73104, USA
}

Received July 23, 2017; Accepted December 4, 2017

DOI: 10.3892/or.2017.6160

\begin{abstract}
The aim of the present study was to observe the effects of cytokine signaling suppressor 1 (SOCS1)-silenced dendritic cells (DCs) pulsed with epidermal growth factor receptor (EGFR) fusion protein on the activation of $\mathrm{T}$ lymphocyte and cytotoxic T-lymphocyte (CTL) activity against Hep-2 cells. DCs were derived from the medullary cells of mice and authenticated by flow cytometry (FCM). Recombinant glutathione- $S$-transferase (GST)-EGFR fusion protein was produced and purified. After being pulsed with it, DCs were modified by recombinant SOCS1-siRNA adenoviral to silence SOCS1 gene expression. The maturation of DCs was evaluated by FCM. The effects of modified DCs on T-cell proliferation were assessed by MTT assay. The killing effects against Hep-2 cells of CTL were assessed by lactate dehydrogenase (LDH) release assay. High-purity DCs from the medullary cells of mice were obtained. Compared with the control, EGFR-pulsed DCs displayed higher expression of cell surface molecules, including CD83, CD860 and HLA-DR. The MTT assay revealed that all of the EGFR-pulsed, SOCS1-silenced and EGFR-pulsed plus SOCS1-silenced DCs had an enhanced capacity to stimulate T-lymphocyte proliferation. As expected, EGFR-pulsed plus SOCS1-silenced DCs had the strongest effects on T-cell proliferation. The splenic T cells isolated from both EGFR-pulsed DC-immunized mice and EGFR-pulsed plus SOCS1-silenced DC-immunized mice enhanced the cytotoxicity against Hep-2 cells, while T cells from EGFR-pulsed plus SOCS1-silenced DC-immunized mice exhibited significantly higher cytotoxicity than those from EGFR-DC-immunized mice. The EGFR-pulsed SOCS1-siRNA-silenced DCs had the strongest effects on activation of T-cell proliferation and the CTL activity against Hep-2 cells.
\end{abstract}

Correspondence to: Professor Xuefeng Wang, Department of Otolaryngology Head and Neck Surgery, Liaoning Medical University, 40 Songpo Road, Jinzhou, Liaoning 121000, P.R. China

E-mail: wangxf121@126.com

Key words: dendritic cells, SOCS1, EGFR, Hep-2 cell,immunotherapy

\section{Introduction}

Head and neck squamous cell carcinoma (HNSCC) describes a broad range of tumors that arise from the base of the skull to the clavicles. The global incidence of HNSCC exceeds half a million annually, making it the fifth most common cancer worldwide (1). Surgery resection is the classical treatment for HNSCC, with or without chemoradiation. Despite advances in surgical techniques and the institution of novel chemoradiation approaches, little improvement has been achieved on the 5-year survival rate over the past 30 years (2). Current treatment of HNSCC has limited effectiveness in improving the survival rate, and immunotherapy may be a promising strategy.

Epidermal growth factor receptor (EGFR) is a $170-\mathrm{kDa}$ transmembrane growth-regulating glycoprotein, which has an intrinsic tyrosine-specific kinase activity.Ligand binding toEGFR induces receptor dimerization and causes autophosphorylation and/or cross-phosphorylation of several tyrosine residues, which in turn initiate an intracellular signaling cascade, ultimately resulting in increased proliferation and differentiation (3). Overexpression of EGFR has been frequently observed in many malignancies, such as HNSCC, non-small cell lung, breast, colon and pancreatic cancer (4-8). The level of EGFR expression is believed to be associated with nodal status and prognosis of these patients (9). Therefore, EGFR is considered to be an attractive molecular target for cancer therapeutics. EGFR monoclonal antibodies (mABs) and small-molecule EGFR tyrosine kinase inhibitors (TKIs) are 2 major therapeutic agents that target EGFR and have been demonstrated to be effective in prolonging survival in HNSCC patients (10). Dendritic cells (DCs), which are the most potent and competent antigen-presenting cells (APCs), have the unique capability of sensitizing naive $\mathrm{T}$ cells to protein antigens. The cytotoxic T lymphocyte (CTL) responses elicited by DCs can kill the tumor cells directly, whereas the mAbs and TKIs inhibit tumor growth mainly by blocking the EGFR signal-transduction pathway. The ability of DCs to present tumor antigens and thereby generate tumor-specific immunity has been demonstrated in several clinical trials (11-13). A previous study indicated that glutathione- $S$-transferase (GST)-EGFR pulsing DCs could effectively elicit CTL activity and prevent tumor progression in animal models (11).

Another possibility to enhance the potency of DC-based immunotherapy is to silence the negative immunoregulatory 
pathways. Cytokine signaling suppressor 1 (SOCS1) is a key regulator of cytokine signaling that is important for maintaining the balance of immune responses. This signaling pathway also plays important roles in DC maturation through its negative cytokine signaling feedback loops. SOCS1 has been discovered as a critical inhibitory molecule in cytokine response and antigen presentation by DCs, regulating the magnitude of both innate and adaptive immunity (14). Previous studies have suggested that SOCS1-deficient DCs induced higher naive T cell activation (15) and stronger Th1-type responses both in vitro and in vivo in inflammatory disease and systemic autoimmunity (16). Reducing the expression of SOCS1 facilitated an effective immune response against HNSCC (17).

In the present study, we postulated that immunotherapy using SOCS1-silenced DCs pulsed with EGFR may be an effective approach and provide a novel strategy for HNSCC treatment. In the present study, SOCS1-silenced DCs pulsed with the GST-EGFR fusion protein were used to trigger CTL activity in a mouse model, and the preventive and therapeutic antitumor effects on Hep-2 cells were observed in vitro by a lactate dehydrogenase $(\mathrm{LDH})$ release assay.

\section{Materials and methods}

Cell culture. The Hep-2 human laryngeal carcinoma cell line, was obtained from the Chinese PLA General Hospital. Cells were cultured in RPMI-1640 (Gibco Life Technologies, Carlsbad, CA, USA), supplemented with $10 \%$ fetal bovine serum (FBS), $100 \mu \mathrm{g} / \mathrm{ml}$ penicillin and $100 \mu \mathrm{g} / \mathrm{ml}$ streptomycin (all obtained from GE Healthcare Life Sciences, Logan, UT, USA) in humidified $5 \% \mathrm{CO}_{2}$ at $37^{\circ} \mathrm{C}$. Trypsin solution $(0.25 \%$; GE Healthcare Life Sciences) was used to detach cells from the culture flask. Culture medium was changed every 2 days.

Animals. Male SD mice were purchased from the Experimental Center of Yangzhou University (Jiangsu, China) and housed in the Central Animal Facility at Liaoning Medical College. All the mice were aged 6-8 weeks at the start of the investigation and their body weight was in the range of 160-180 g. The animals were acclimated for at least 1 week before any of the experiments were undertaken. All studies involving mice were approved by the Institute's Animal Care and Use Committee (Liaoning Provincial Science and Technology Department).

Construction of the expression vector encoding the extracellular domain (ECD) of EGFR. The EGFR-wt plasmid was obtained from Addgene (Cambridge, CA, USA). The plasmid corresponding to the ECD of EGFR was amplified by reverse transcription polymerase chain reaction (RT-PCR). The upstream primer, 3'-gCTGGAGGAAAAGAAAGTTTGCCA AGG-5' includes a SmaI excision site. The downstream primer, 3'-GGGGACGGGATCTTAGGCCC-5' contains a SmaI excision site and a stop codon. Total RNA was reversetranscribed using the specific downstream primer. PCR cycle parameters were $95^{\circ} \mathrm{C}$ for $30 \mathrm{sec}, 66^{\circ} \mathrm{C}$ for $30 \mathrm{sec}$, and $72^{\circ} \mathrm{C}$ for $90 \mathrm{sec}$, for a total 30 cycles, followed by a $10 \mathrm{~min}$ final extension at $72^{\circ} \mathrm{C}$. The RT-PCR product, a 2-kb fragment, was cloned into the pGEM-T easy vector for sequence analysis. The fragment encoding for the ECD of EGFR was recovered using SmaI enzymes and cloned into SmaI sites of the
pGEX-4T-2 expression vector, generating the pGEX-4T-2EGFR plasmid.

Expression and purification of GST-EGFR fusion and GST proteins. The pGEX-4T-2-EGFR and pGEX-4T-2 plasmids were transformed into Escherichia coli $(E$. coli) XL1-Blue, respectively, and the expression of GST-EGFR fusion or GST proteins was induced with $0.1 \mathrm{mM}$ isopropyl $\beta$-D-thiogalactoside (IPTG) at $23^{\circ} \mathrm{C}$ overnight. The GST fusion protein used in the present study was to facilitate the expression of E. coli and purification of the recombinant protein. The expression of the proteins was identified by sodium dodecyl sulfate-polyacrylamide gel electrophoresis (SDS-PAGE) and protein immunoblots (western blotting) with an anti-GST antibody. The MagneGST Protein Purification System was used to purify the soluble GST-EGFR fusion and GST proteins. The proteins were eluted with buffer $(50 \mathrm{mM}$ glutathione, $\mathrm{pH}$ 7.0-8.0; $50 \mathrm{mM}$ Tris- $\mathrm{HCl}, \mathrm{pH} 8.1$ ) and the purified proteins were examined by SDS-PAGE.

Generating DCs. Bone marrow-derived DCs were isolated and maintained as previously described with minor modifications (18). Briefly, bone marrow cells were obtained from femurs and tibias of SD mice and filtered through a nylon mesh. Red blood cells (RBC) were depleted with lysis buffer and washed with phosphate buffered-saline (PBS) twice. Then, the cells were seeded at $2 \times 10^{6}$ cells $/ 100-\mathrm{mm}$ dish in RPMI-1640 medium supplemented with $10 \%$ FBS and $500 \mathrm{U} / \mathrm{ml} \mathrm{rmGM-CSF}, 500 \mathrm{U} / \mathrm{ml} \mathrm{rmIL-4}$ and $500 \mathrm{U} / \mathrm{ml}$ TNF- $\alpha$ at $37^{\circ} \mathrm{C}$ and $5 \% \mathrm{CO}_{2}$. On days 4,6 and 7, half of the culture supernatants were collected and centrifuged, respectively. Each cell pellet was re-suspended in $5 \mathrm{ml}$ of a fresh RPMI-1640 medium containing $500 \mathrm{U} / \mathrm{ml} \mathrm{rmGM-CSF}$, rmIL-4 and TNF- $\alpha$ and returned to the original plate. On day 7 , the DCs were harvested for subsequent experiments.

Synthesis of the siSOCS1 gene and gene transfection. The siRNA molecules used for suppression of the murine SOCS1 gene and the negative control siRNA (which does not target any sequence present in the murine genome) were all obtained from Biosci Co., Ltd. (Hangzhou, China). Recombinant adenovirus expression vector GV248 was used as the vector for SOCS1-siRNA. Transient transfection of siRNA was carried out with Lipofectamine 2000 reagent (Invitrogen, Waltham, MA, USA) according to the manufacturer's instructions.

Quantitative RT-PCR. The relative expression of SOCS1 mRNA in transfected DCs was evaluated by quantitative real-time PCR (qRT-PCR). Total RNA was prepared using TRIzol reagent (Invitrogen) according to the manufacturer's instructions. cDNA was synthesized with $1 \mu \mathrm{g}$ of total RNA by reverse transcriptase (Takara, Shiga, Japan). For quantitative determination of SOCS1 expression, qRT-PCR analysis was performed with LightCycler (Roche Diagnostics, Basel, Switzerland).

Western blot analysis. In western blot analysis, cell lysates were separated by SDS-polyacrylamide gel electrophoresis, transferred onto polyvinylidene fluoride (PVDF) membranes, and probed with a rabbit monoclonal anti-SOCS1 antibody (cat. 


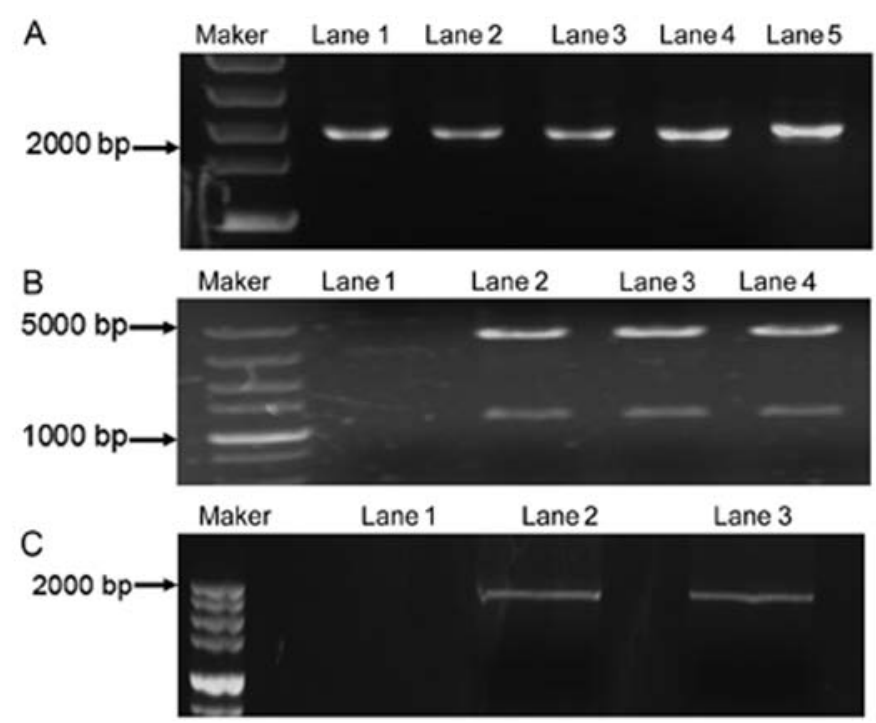

Figure 1. Construction of the expression vector encoding EGFR-ECD. (A) EGFR-ECD cDNA was obtained by RT-PCR. Lanes 1-5, EGFR-ECD cDNA. (B) The EGFR/pGEX-4T-2 plasmid was ascertained by SmaI digestion. Lane 1, blank control; lanes 2-4, digested EGFR/pGEX-4T-2 plasmid. (C) The product was separated by $1 \%$ agarose, and 2 expected fragments appeared. EGFR, epidermal growth factor receptor; ECD, extracellular domain; RT-PCR, reverse transcription polymerase chain reaction.

no. 10-P1074; ARP) was reconstitutioned with distilled water. Bound antibodies were detected using a horseradish peroxidase (HRP)-labeled goat anti-rabbit IgG (cat. no. BG08T-1; Genemark Technology Co., Ltd., Tainan, Taiwan), and then were developed using 3,3',5,5'-tetramethylbenzidine (TMB; cat. no. 1215-100; BioVision, Inc., Milpitas, CA, USA).

Pulsing DCs and flow cytometric analysis. After adenovirus SOCS1 transfection for $4 \mathrm{~h}, \mathrm{DC}$ were incubated with GST-EGFR fusion protein $(20,50$ and $100 \mu \mathrm{g} / \mathrm{ml})$ for $12 \mathrm{~h}$. As a control, unpulsed DCs were also cultured for $12 \mathrm{~h}$. Then, DCs were collected, and the expression of surface molecules on DCs was quantified by flow cytometry using FITC- or PE-conjugated Ab (anti-CD83, anti-HLA-DR and anti-CD86). The samples were analyzed using a FACSCalibur flow cytometer and CellQuest software (BD Biosciences, Franklin Lakes, NJ, USA).

T-cell proliferation assays. T lymphocytes were isolated from the spleens of SD mice by negative immunomagnetic selection using a Ficoll-Hypaque kit (GE Healthcare Life Sciences, Buckinghamshire,UK).For the proliferation assays, Tcells were seeded into $96-w e l l$ plates at a density of $2 \times 10^{6} / \mathrm{ml}$. The cells were divided into six groups: blank (no cells), control (T cells), DCs (T cells + DCs), EGFR (T cells + EGFR-DCs), SOCS1-silenced ( $\mathrm{T}$ cells + SOCS1-siRNA-DCs), EGFR + SOCS1-silenced ( $\mathrm{T}$ cells + EGFR-SOCS1-siRNA-DCs). T cells were co-cultured with DCs at different DC/T cell ratios (1:5, 1:10 and 1:20) in RPMI-1640 for 3 days at $37^{\circ} \mathrm{C}$ in triplicate. After 3 days of incubation, T-cell proliferation status was examined by MTT assay. Cells were incubated at $37^{\circ} \mathrm{C}$ for $4 \mathrm{~h}$ following the addition of $20 \mu \mathrm{l}$ MTT to each well and the absorbance at $490 \mathrm{~nm}$ was detected using a microplate reader.

Cytotoxic T lymphocyte assay. For the cytotoxic T lymphocyte (CTL) assay, $1 \times 10^{6}$ Hep-2 cells were subcutaneously injected into the left flanks of 6-week-old SD mice. After 10 days, the mice that had a mean tumor size of $10 \mathrm{~mm}$ were chosen for the following experiments. The mice were divided into 3 groups, and were subcutaneously injected into the right flanks with untreated DCs, EGFR-pulsed DCs and EGFR-SOCS1 silenced pulsed DCs, respectively. After 7 days, T cells were separated and enriched from each group of mice as previously described. Hep- 2 cells were seeded in 96 -well plates at a density of $2 \times 10^{6} \%$ $\mathrm{ml}$. The Hep-2 cells were divided into 6 groups: experimental (Hep-2 cells plus T cells from DCs, EGFR-DCs and EGFR plus SOCS1-silenced DC mice), blank (no cells), spontaneous (Hep-2 cells only) and the maximum group (Hep-2 cells plus cell lysis buffer). Then, Hep-2 cells were co-cultured with $\mathrm{T}$ cells at different T/Hep-2 cell ratios (1:25, 1:50 and 1:100) in RPMI-1640 for $30 \mathrm{~min}$ at $37^{\circ} \mathrm{C}$ in triplicate. After $30 \mathrm{~min}$ of incubation, samples of the cultured wells were then harvested and the cytotoxicity of the T cells against Hep-2 cells was determined using an LDH activity assay kit. Wavelength (450 nm) absorbance data were collected using a standard 96-well plate reader. The percentage of specific lysis was calculated as: (experimental - spontaneous)/(maximum - spontaneous) $\mathrm{x} 100 \%$.

Statistical analysis. Data are presented as the means \pm SD. The significance of differences between the values of different groups was evaluated by Student's t-test or ANOVA test, and $\mathrm{p}<0.05$ was considered to indicate a statistically significant result. SPSS 21.0 software was used for these analyses.

\section{Results}

Construction of the expression vector encoding the ECD of EGFR and GST-EGFR fusion protein expression and purification. The EGFR-wt plasmid was used as a template for EGFR-ECD cDNA expression. The RT-PCR product was separated by $1 \%$ agarose gel electrophoresis and stained with ethidium bromide. The expected length was $1.6 \mathrm{kbp}$ (Fig. 1A). The sequence of the ECD of EGFR was confirmed, by dideoxynucleotide sequencing analysis, to be 

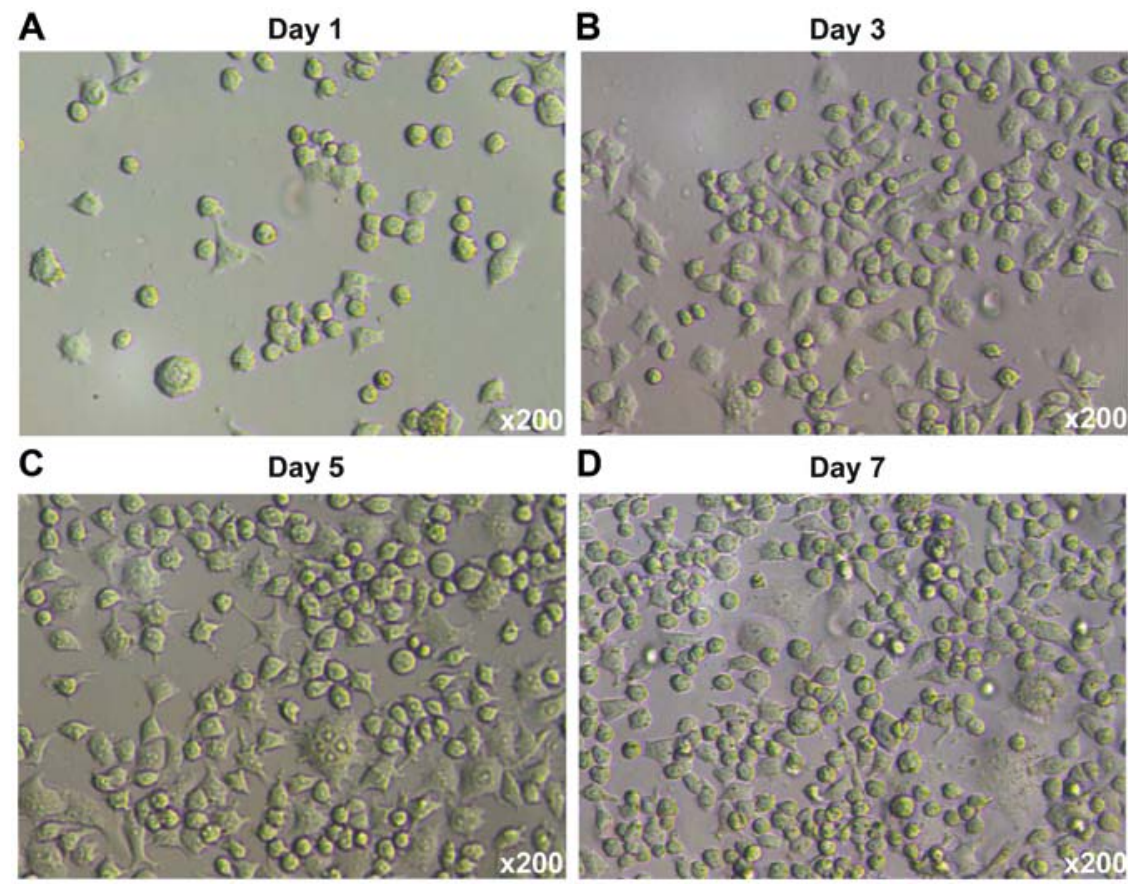

Figure 2. Phase contrast micrographs illustrating the development and isolation of dendritic cells from liquid cultures of mice bone marrow cells supplemented with GM-CSF, rmIL-4 and TNF- $\alpha$ after (A) 1, (B) 3, (C) 5 and (D) 7 days of culture respectively; magnification, x200.
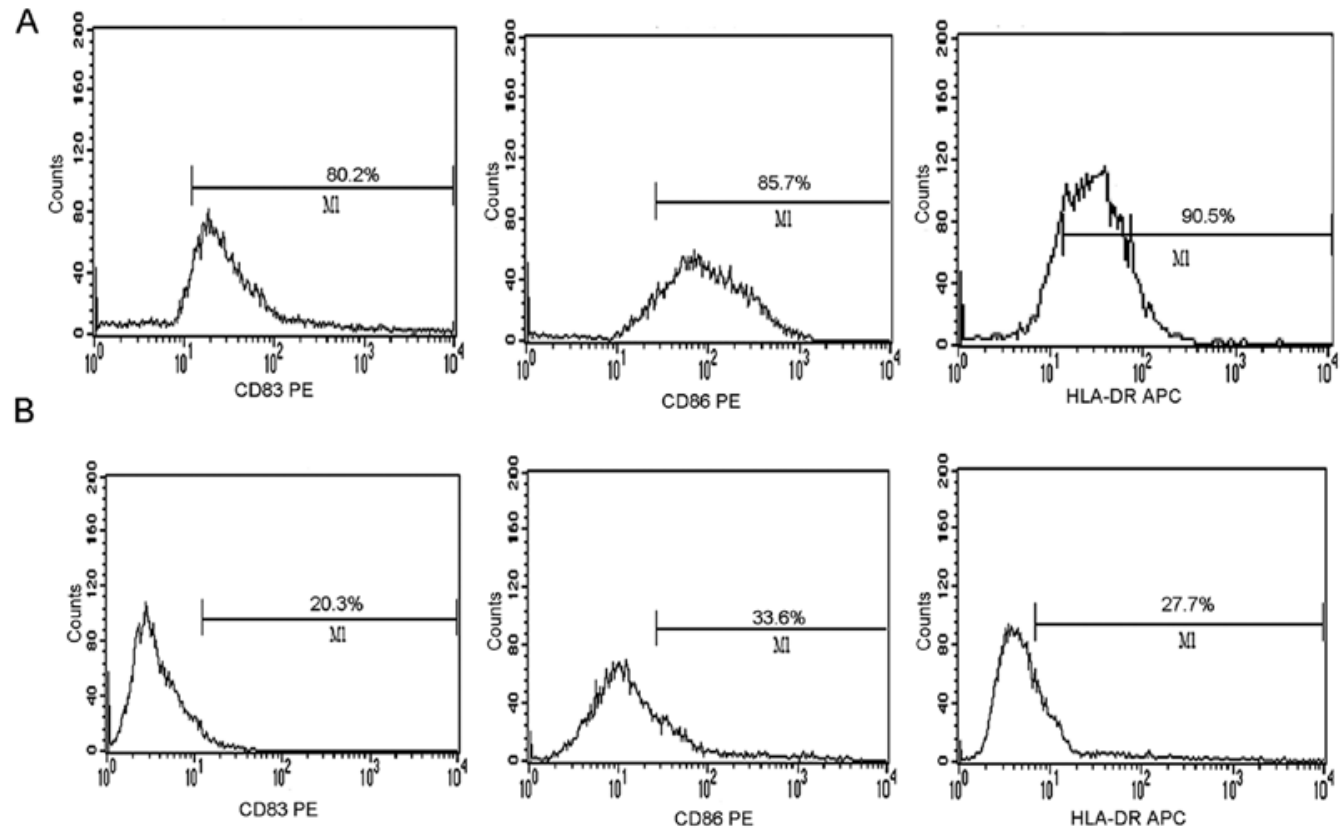

Figure 3. DCs mature when co-cultured with proteins. Expression levels of HLA-DR, CD86 and CD83 with (A) GST-EGFR-pulsed on the DC surfaces were higher than those on (B) the unpulsed DC surfaces. DCs, dendritic cells; GST, glutathione-S-transferase; EGFR, epidermal growth factor receptor.

identical to those previously reported (data not shown) (19). Then, the EGFR-ECD cDNA was successfully cloned into the pGEX-4T-2 expression vector as previously described. The EGFR-ECD expression was reconfirmed by the RT-PCR (Fig. 1B). The pGEX-4T-2-EGFR plasmid was established by SamI digestion. The product was separated by $1 \%$ agarose, and 2 expected fragments appeared (Fig. 1C). The expression of the soluble GST-EGFR or GST was analyzed by SDS-PAGE and western blotting. The proteins were purified by the MagneGST Protein Purification System and confirmed by SDS-PAGE, revealing the protein bands again (data not shown). The purity of the acquired proteins was $>90 \%$.

Preparation, sensitization and characterization of DCs. Bone marrow-derived DCs were isolated from SD mice and maintained as previously described (20). The DCs were cultured in media supplemented with GM-CSF, rmIL-4 and TNF- $\alpha$ for 7 days. On day 7, the DCs were harvested for subsequent experiments. The phase contrast micrographs illustrating the development and isolation of DCs are shown in Fig. 2. For DC 


\section{A}

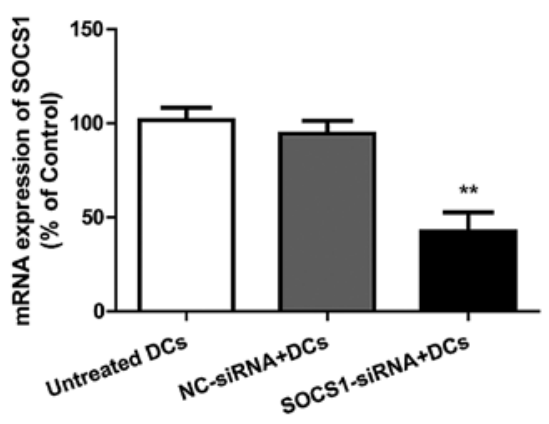

B

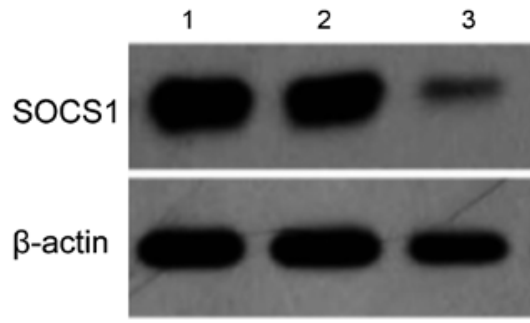

1 Untreated DCs

2 Negative control siRNA+DCs

3 SOCS1-SiRNA+DCs

Figure 4. Ablation of SOCS1 expression on DCs. (A) RT-PCR and (B) western blotting indicated that the expression of SOCS1 was significantly knocked down after adenovirus SOCS1-siRNA transfection. The mean \pm SD of data from 3 experiments is presented $\left(^{* *} \mathrm{p}<0.01\right.$, compared with the NC-siRNAtreated group). SOCS1, cytokine signaling suppressor 1; DCs, dendritic cells; RT-PCR, reverse transcription polymerase chain reaction.

sensitization, the DCs were incubated with GST-EGFR fusion protein at different concentrations. The degree of expression of CD83, CD86 and HLA-DR on the DC surfaces was evaluated by flow cytometry after a 48 -h stimulation with proteins. The positive cells among the DCs pulsed with GST-EGFR were 80.2, 85.7 and $90.5 \%$, respectively. These results were greater than those of the DCs unpulsed with protein, in which the positive cells were 20.3, 33.6 and 27.7\%, respectively (Fig. 3A and B).

SOCS1 silencing effectively enhances T-lymphocyte proliferation. The SOCS1 gene is an important regulator that suppresses DC maturation and cytokine production during inflammatory response. To clarify the ability of $\mathrm{T}$ cell differentiation under SOCS1 gene silencing, we compared the proliferation of $\mathrm{T}$ cells after incubation with SOSC1-siRNA DCs or negative control DCs after being pulsed with EGFR fusion protein by MTT assay. The results in Fig. 4 indicated that the mRNA and protein expression of SOCS1 in DCs were knocked down by 50-70\%. As shown in Fig. 5, all of the EGFR-pulsed DCs, SOCS1-silenced DCs and EGFR plus SOCS1-silenced DCs had an enhanced capacity to stimulate the proliferation of $\mathrm{T}$ lymphocytes at $\mathrm{DC} / \mathrm{T}$ cell ratios of 1:5 and 1:10. As expected, EGFR plus SOCS1-silenced DCs had the strongest effects on $\mathrm{T}$ cell proliferation. At the DC/T cell ratio of 1:20, only EGFR plus SOCS1-silenced DCs enhanced T-cell proliferation while EGFR-pulsed and SOCS1-silenced DCs had no effect on T-cell proliferation.

EGFR plus SOCS1-silenced DC immunization effectively enhances cytotoxic T-lymphocyte activity against Hep-2

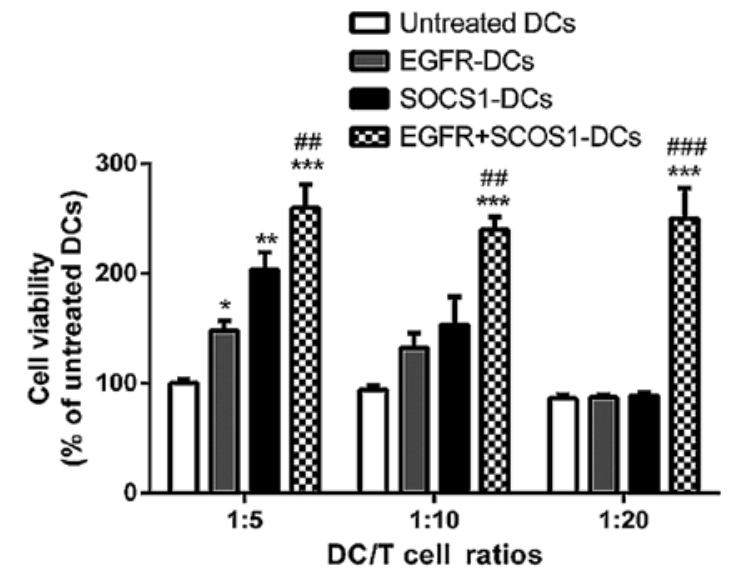

Figure 5. SOCS1-siRNA effectively enhances T-lymphocyte proliferation. MTT assays indicated that DCs treated with EGFR, SOCS1-siRNA and EGFR plus SOCS1-siRNA have enhanced capacity to activate T-cell proliferation at different DC/T cell ratios, including 1:5, 1:10 and 1:20. The mean \pm SD of data from 3 experiments is shown. $\left({ }^{*} \mathrm{p}<0.05,{ }^{* *} \mathrm{p}<0.01,{ }^{* * *} \mathrm{p}<0.001\right.$, compared with the untreated DC groups; ${ }^{\# \#} \mathrm{p}<0.01,{ }^{\# \# \#} \mathrm{p}<0.001$, compared with the EGFR-DC groups). SOCS1, cytokine signaling suppressor 1; DCs, dendritic cells; EGFR, epidermal growth factor receptor.

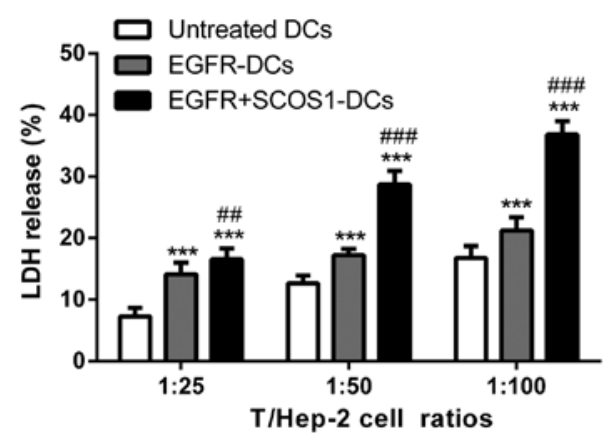

Figure 6. SOCS1-siRNA effectively enhances cytotoxic T-lymphocyte activity against Hep-2 cells. Cytotoxicity of the T cells isolated from each group against Hep-2 cells was assessed by an LDH release assay at different T/Hep-2 cell ratios including 1:25, 1:50 and 1:100. T cells derived from EGFR + SOCS1-siRNA DC-immunized mice exhibited significantly higher cytotoxicity than that of cells from untreated DCs or EGFR-pulsed DC-immunized mice. The mean \pm SD of the data from twelve experiments is presented $\left({ }^{* * *} \mathrm{p}<0.001\right.$, compared with the untreated DC groups; ${ }^{\# \#} \mathrm{p}<0.01$,

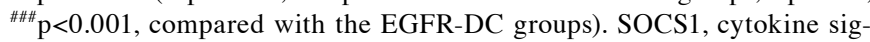
naling suppressor 1; LDH, lactose dehydrogenase; DCs, dendritic cells; EGFR, epidermal growth factor receptor.

cells. A CTL assay was performed at different T/Hep-2 cell ratios of 1:25, 1:50 and 1:100. The splenic $\mathrm{T}$ cells isolated from both EGFR-pulsed DC-immunized mice and EGFR plus SOCS1-silenced DC-immunized mice enhanced the cytotoxicity against Hep-2 cells, while T cells from EGFR plus SOCS1-silenced DC-immunized mice exhibited significantly higher cytotoxicity than those from EGFR-DC-immunized mice (Fig. 6).

\section{Discussion}

The development of cancer immunotherapy has led to great clinical advances and provided a new weapon against cancer (21). The prerequisite of immunotherapy is to identify an efficient tumor-specific antigen. EGFR is a tumor-associated 
antigen of HNSCC. More than $80 \%$ of head and neck tumors overexpress EGFR (22). The knowledge that EGFR is overexpressed in the majority of HNSCC cells provides a rationale for the use of anti-EGFR therapies $(23,24)$. Cetuximab, a chimeric monoclonal antibody (mAb) targeting the extracellular portion of EGFR, was found to enhance survival when combined with radiotherapy in patients with advanced HNSCC (25). Therefore, EGFR is an ideal target in antitumor immunotherapy of HNSCC. Dendritic cells (DCs) are the most potent antigen-presenting cells in the immune system and have the unique ability to take up and efficiently present antigens to naive T lymphocytes. DCs can also interact with B cells and natural killer (NK) cells, thus bridging the gap between innate and adaptive immunities. DC vaccinations have been demonstrated to be safe and efficient in inducing the expansion of circulating CTLs that are specific for tumor antigens (26). Thus, in the present study, we pulsed DCs with a GST-EGFR fusion protein in order to provoke the specific immune response targeted against EGFR and HNSCC.

Several cancer vaccine studies have suggested that the therapeutic vaccination outcome (success or failure) is correlated with the vaccine-induced expansion of antigen-specific effector T cells $(27,28)$. In the present study, we compared EGFR-pulsed DCs with control DCs and found that the former displayed even higher expression of cell surface molecules, such as CD83, CD860 and HLA-DR (which are common indicators of the maturation of DCs). Effective therapeutic antitumor activities of the EGFR-pulsed DC vaccine against SCC tumor cells were confirmed in a previous study (29). Similar to the results of previous studies, EGFR fusion protein-immunized DCs had the strongest effects on activated T-cell differentiation. Meanwhile, the strongest CTL response against Hep-2 cells was found in the group immunized with EGFR-pulsed DCs compared with the control groups. CTLs are believed to be critical effectors of antitumor immune responses (30), whereas the $\mathrm{CD}^{+}$ $\mathrm{T}$ cells, characterized by the secretion of IFN- $\gamma$, are primarily responsible for activating and regulating the development and persistence of CTLs (31). Previous studies in mice revealed that the in vivo induction of CTL responses, particularly those induced through cross-priming of exogenous antigens by DCs, is dependent on a CD4 $4^{+} \mathrm{T}$-cell response $(32,33)$. Moreover, $\mathrm{CD} 4^{+}$ $\mathrm{T}$ cells are also essential for the activation of memory CTLs into tumor killer cells (34). Therefore, our in vitro observations indicated that GST-EGFR-pulsed DCs induced effective therapeutic and preventive antitumor immunity against Hep-2 cells.

In addition to the expression of co-stimulatory molecules that facilitate an immune response, DCs are also equipped with negative feedback mechanisms that control their cytokine function. According to previous literature, the SOCS1 gene is an important regulator that suppresses DC maturation and cytokine production during inflammatory response. In the present study, we demonstrated that a SOCS1-siRNA approach can be used to silence negative regulatory molecules in DCs and thereafter to modulate immune response. Although, the abolition of SOCS1 in DCs could by itself induce the expression of co-stimulatory molecules as suggested by other studies $(35,36)$, we found that there was no significant difference in surface molecule expression between EGFR plus SOCS1-silenced DCs and only EGFR-pulsed DCs (data not shown). Ablation of SOCS1 in antigen-presenting cells has been reported to enhance cellular immune and subsequent cytokine responses in mice when they are challenged by a pathogen (37). Our data revealed that reversing the immunity-attenuating mechanism of SOCS1 activated the function of DCs, including the promotion of DC maturation and activation of T-cell differentiation. Concurrently, SOCS1-siRNA-treated DCs also mediated innate immunity by possessing a stronger CTL activity against Hep-2 cells. Compared to the EGFR-pulsed only DCs, EGFR plus SOCS1-silenced DCs had a stronger effect on T-cell proliferation and CTL against Hep-2 cells. These results revealed that the targeted modulation of SOCS1 expression in DCs could be exploited as a novel molecular adjunct to improve the potency of vaccine-induced T cells against HNSCC.

In summary, the present study revealed that SOCS1 silencing can be used to silence immunosuppressive molecules in GST-EGFR pulsed DCs, enhance the T-cell proliferation and the CTL activity against Hep-2 cells in vitro. This provides a promising approach for increasing antitumor activity of EGFR-targeted therapy of HNSCC.

\section{Acknowledgements}

The present study was funded by the Youth Foundation of the First Affiliated Hospital of Liaoning Medical University and the Liaoning Science and Technology Project (2012225019).

\section{References}

1. Jemal A, Bray F, Center MM, Ferlay J, Ward E and Forman D: Global cancer statistics. CA Cancer J Clin 61: 69-90, 2011.

2. Mao L, Hong WK and Papadimitrakopoulou VA: Focus on head and neck cancer. Cancer Cell 5: 311-316, 2004.

3. Yarden Y: The EGFR family and its ligands in human cancer. signalling mechanisms and therapeutic opportunities. Eur J Cancer 37 (Suppl 4): S3-S8, 2001.

4. Salomon DS, Brandt R, Ciardiello F and Normanno N: Epidermal growth factor-related peptides and their receptors in human malignancies. Crit Rev Oncol Hematol 19: 183-232, 1995.

5. Woodburn JR: The epidermal growth factor receptor and its inhibition in cancer therapy. Pharmacol Ther 82: 241-250, 1999.

6. Wiley HS: Trafficking of the ErbB receptors and its influence on signaling. Exp Cell Res 284: 78-88, 2003.

7. Nicholson RI, Gee JM and Harper ME: EGFR and cancer prognosis. Eur J Cancer 37 (Suppl 4): S9-S15, 2001.

8. Herbst RS: Targeted therapy in non-small-cell lung cancer. Oncology 16 (Suppl 9): 19-24, 2002.

9. Galanis E, Buckner J, Kimmel D, Jenkins R, Alderete B, O'Fallon J, Wang CH, Scheithauer BW and James CD: Gene amplification as a prognostic factor in primary and secondary high-grade malignant gliomas. Int J Oncol 13: 717-724, 1998.

10. Kabolizadeh P, Kubicek GJ, Heron DE, Ferris RL and Gibson MK: The role of cetuximab in the management of head and neck cancers. Expert Opin Biol Ther 12: 517-528, 2012.

11. Celluzzi CM, Mayordomo JI, Storkus WJ, Lotze MT and Falo LD Jr: Peptide-pulsed dendritic cells induce antigen-specific CTL-mediated protective tumor immunity. J Exp Med 183: 283-287, 1996

12. Mayordomo JI, Zorina T, Storkus WJ, Zitvogel L, Celluzzi C, Falo LD, Melief CJ, Ildstad ST, Kast WM, Deleo AB, et al: Bone marrow-derived dendritic cells pulsed with synthetic tumour peptides elicit protective and therapeutic antitumour immunity. Nat Med 1: 1297-1302, 1995.

13. Fong L, Brockstedt D, Benike C, Breen JK, Strang G, Ruegg CL and Engleman EG: Dendritic cell-based xenoantigen vaccination for prostate cancer immunotherapy. J Immunol 167: 7150-7156, 2001.

14. Kubo M, Hanada T and Yoshimura A: Suppressors of cytokine signaling and immunity. Nat Immunol 4: 1169-1176, 2003.

15. Hanada T, Yoshida H, Kato S, Tanaka K, Masutani K, Tsukada J, Nomura Y, Mimata H, Kubo M and Yoshimura A: Suppressor of cytokine signaling-1 is essential for suppressing dendritic cell activation and systemic autoimmunity. Immunity 19: 437-450, 2003. 
16. Hanada T, Tanaka K, Matsumura Y, Yamauchi M, Nishinakamura H, Aburatani H, Mashima R, Kubo M, Kobayashi T and Yoshimura A: Induction of hyper Th1 cell-type immune responses by dendritic cells lacking the suppressor of cytokine signaling-1 gene. J Immunol 174: 4325-4332, 2005.

17. Shi D, Li D, Yin Q, Qiu Y, Yan H, Shen Y, Lu G and Liu W: Silenced suppressor of cytokine signaling 1 (SOCS1) enhances the maturation and antifungal immunity of dendritic cells in response to Candida albicans in vitro. Immunol Res 61: 206-218, 2015.

18. Lutz MB, Kukutsch N, Ogilvie AL, Rössner S, Koch F, Romani N and Schuler G: An advanced culture method for generating large quantities of highly pure dendritic cells from mouse bone marrow. J Immunol Methods 223: 77-92, 1999.

19. Reiter JL, Threadgill DW, Eley GD, Strunk KE, Danielsen AJ, Sinclair CS, Pearsall RS, Green PJ, Yee D, Lampland AL, et al: Comparative genomic sequence analysis and isolation of human and mouse alternative EGFR transcripts encoding truncated receptor isoforms. Genomics 71: 1-20, 2001.

20. Gabrilovich DI, Nadaf S, Corak J, Berzofsky JA and Carbone DP Dendritic cells in antitumor immune responses. II. Dendritic cells grown from bone marrow precursors, but not mature DC from tumor-bearing mice, are effective antigen carriers in the therapy of established tumors. Cell Immunol 170: 111-119, 1996.

21. Sharma P and Allison JP: The future of immune checkpoint therapy. Science 348: 56-61, 2015.

22. Herbst RS and Shin DM: Monoclonal antibodies to target epidermal growth factor receptor-positive tumors: A new paradigm for cancer therapy. Cancer 94: 1593-1611, 2002.

23. Sharafinski ME, Ferris RL, Ferrone S and Grandis JR: Epidermal growth factor receptor targeted therapy of squamous cell carcinoma of the head and neck. Head Neck 32: 1412-1421, 2010

24. Ferris RL, Jaffee EM and Ferrone S: Tumor antigen-targeted, monoclonal antibody-based immunotherapy: Clinical response, cellular immunity, and immunoescape. J Clin Oncol 28 . 4390-4399, 2010.

25. Bonner JA, Harari PM, Giralt J, Azarnia N, Shin DM, Cohen RB, Jones CU, Sur R, Raben D, Jassem J, et al: Radiotherapy plus cetuximab for squamous-cell carcinoma of the head and neck. N Engl J Med 354: 567-578, 2006.

26. Palucka $\mathrm{K}$ and Banchereau J: Cancer immunotherapy via dendritic cells. Nat Rev Cancer 12: 265-277, 2012.
27. Paczesny S, Banchereau J, Wittkowski KM, Saracino G, Fay J and Palucka AK: Expansion of melanoma-specific cytolytic $\mathrm{CD}^{+} \mathrm{T}$ cell precursors in patients with metastatic melanoma vaccinated with $\mathrm{CD} 34^{+}$progenitor-derived dendritic cells. J Exp Med 199: 1503-1511, 2004.

28. Welters MJ, Kenter GG, de Vos van Steenwijk PJ, Löwik MJ, Berends-van der Meer DM, Essahsah F, Stynenbosch LF, Vloon AP, Ramwadhdoebe TH, Piersma SJ, et al: Success or failure of vaccination for HPV16-positive vulvar lesions correlates with kinetics and phenotype of induced T-cell responses. Proc Natl Acad Sci USA 107: 11895-11899, 2010.

29. Yang BB, Jiang H, Chen J, Zhang X, Ye JJ and Cao J: Dendritic cells pulsed with GST-EGFR fusion protein: Effect in antitumor immunity against head and neck squamous cell carcinoma. Head Neck 32: 626-635, 2010.

30. Melief CJ: Tumor eradication by adoptive transfer of cytotoxic T lymphocytes. Adv Cancer Res 58: 143-175, 1992.

31. Knutson KL and Disis ML: Tumor antigen-specific $\mathrm{T}$ helper cells in cancer immunity and immunotherapy. Cancer Immunol Immunother 54: 721-728, 2005.

32. Bennett SR, Carbone FR, Karamalis F, Miller JF and Heath WR: Induction of a $\mathrm{CD} 8^{+}$cytotoxic $\mathrm{T}$ lymphocyte response by cross-priming requires cognate $\mathrm{CD} 4^{+} \mathrm{T}$ cell help. J Exp Med 186: 65-70, 1997.

33. Schoenberger SP, Toes RE, van der Voort EI, Offringa R and Melief CJ: T-cell help for cytotoxic T lymphocytes is mediated by CD40-CD40L interactions. Nature 393: 480-483, 1998.

34. Gao FG, Khammanivong V, Liu WJ, Leggatt GR, Frazer IH and Fernando GJ: Antigen-specific $\mathrm{CD}^{+} \mathrm{T}$-cell help is required to activate a memory $\mathrm{CD} 8^{+} \mathrm{T}$ cell to a fully functional tumor killer cell. Cancer Res 62: 6438-6441, 2002.

35. Kobayashi T and Yoshimura A: Keeping DCs awake by putting SOCS1 to sleep. Trends Immunol 26: 177-179, 2005.

36. Hu Q, Qin X, Qian G, Jiang S, Li H, Jiang M, Li X, Chen SY and Zang YQ: SOCS1 silencing can break high-dose dendritic cell immunotherapy-induced immune tolerance. Mol Med Rep 1: 61-70, 2008

37. Wurtz O, Bajénoff $M$ and Guerder S: IL-4-mediated inhibition of IFN-gamma production by $\mathrm{CD}^{+}{ }^{+} \mathrm{T}$ cells proceeds by several developmentally regulated mechanisms. Int Immunol 16: 501-508, 2004. 